\title{
Preparation of $\mathrm{Ag} / \mathrm{Cu} / \mathrm{Ti}$ Nanofluids by Spark Discharge System and Its Control Parameters Study
}

\author{
Kuo-Hsiung Tseng, ${ }^{1}$ Juei-Long Chiu, ${ }^{1}$ Heng-Lin Lee, ${ }^{1}$ Chih-Yu Liao, ${ }^{1,2}$ \\ Hong-Shiou Lin, ${ }^{1}$ and Yi-Syuan Kao ${ }^{1}$ \\ ${ }^{1}$ Department of Electrical Engineering, National Taipei University of Technology, Taipei 10608, Taiwan \\ ${ }^{2}$ Light Source Division, National Synchrotron Radiation Research Center, Hsinchu 30076, Taiwan \\ Correspondence should be addressed to Kuo-Hsiung Tseng; khtseng@ee.ntut.edu.tw
}

Received 27 December 2014; Accepted 23 February 2015

Academic Editor: Belal F. Yousif

Copyright (C) 2015 Kuo-Hsiung Tseng et al. This is an open access article distributed under the Creative Commons Attribution License, which permits unrestricted use, distribution, and reproduction in any medium, provided the original work is properly cited.

\begin{abstract}
This study selected silver, copper, and titanium as the research objects to explore the relationship between nanofluids properties and electrical discharge machining (EDM) processes. Regarding the products, UV-visible spectroscopy (UV-Vis) was applied to measure the concentration distribution of nanofluids; zeta-size analysis is applied for measuring nanometal particles' Zeta-Potential and the size distribution of metallic particles in the fluid. Finally, various instruments, including scanning electron microscope (SEM), were applied to observe the shape, size, and composition ratio of metal particles after processing. According to the experimental results, the control of the discharge pulse time, in addition to affecting the concentration of metallic liquid and temperature in the process, affects the size of the metal particles after the process. As the resistivity of silver and copper is very low, at about $15 \times 10^{-9} \Omega \cdot \mathrm{m}$, if $T_{\mathrm{ON}}$ is set to between $10 \sim 50 \mu \mathrm{s}$, good preparation efficiency can be obtained. The resistivity of titanium is $420 \times 10^{-9} \Omega \cdot \mathrm{m}$, which is much larger than that of silver or copper. Hence, $T_{\mathrm{ON}}$ should be set to approximately $100 \mu$ s to achieve a good discharge success rate.
\end{abstract}

\section{Introduction}

Previously, in the preparation of metal nanoparticles, nanoparticle size or concentration was controlled chemically by adding suspensions control [1]. In the method proposed by this study, the Electric Spark Discharge Method (ESDM) of EDM is used to crack metal materials into nanometer sized particles. The preparation process requires only deionized water (DW) or an alcohol solution as the medium and setting the parameters including EDM voltage and current to prepare metal nanoparticles suspending in the dielectric liquid.

In the electrical discharge method proposed in this study, the electrical discharge generates an arc discharge between electrodes in the insulating liquid, which then produces a thermal effect on the surfaces of two electrodes. Besides the heat generated by the arc discharge, the processed liquid generates a gasification popping phenomenon due to the discharge pressure and thermal effect, and the melted electrode portion is thus dissolved in the processing liquid with the vaporization of liquid phenomenon [2]. As the electrical discharge is not subject to the hardness, strength, or toughness of the material, any conductive material can be processed. Studies on EDM preparation of nanoparticles in recent years EDM focused on analysis of the characteristics and applications of nanoparticles in dielectric fluid or fluid [3-5]. For example, through arc discharge, the preparation of nanoparticles made of titanium has an excellent corrosion resistance and high strength-weight ratio [6]. Nanoparticles of silver with a good effect on medical sterilization have been successfully developed by EDM methods [7-10]. In the same way, gold nanoparticle fluid is prepared in $95 \%$ alcohol $[11,12]$, with a particle size of about $15-30 \mathrm{~nm}$ and absorption peak wavelength of about $525 \mathrm{~nm}$. This study has found that the suspension of gold nanoparticles in alcohol can be converted into aqueous phase gold nanoparticles by adding the same amount of DW after vaporization of alcohol. After 
phase transformation, the gold particles remain suspended in DW; however, the gold particle size has changed. In the research of $\mathrm{CuO}$ nanoparticles [13] without a suspending agent, $\mathrm{CuO}$ fluid with good stability of suspension can be obtained [14]. The above methods are improved, and the dry vacuum extraction method is applied to prepare $\mathrm{Al}_{2} \mathrm{O}_{3}$ nanoparticles by the vaporization of metal electrodes in the plasma state, combined with a cooling system for water collection [15]. For EDM processing, a set of continuous preparation methods has been developed [16-18], which can effectively be applied to mass production and further developed toward nanotechnology applications.

\section{Materials and Methods}

2.1. Electric Spark Discharge Method. The main materials used in this study were metals (conductors and semiconductors). A variety of parameter settings (voltage/current/onoff duration) were used to observe the spark generation of various metals, in order to determine the characteristic relationships between the processing parameters of three types of metals (silver, titanium, and copper) and nanometal fluids. The purity of the three metals is silver with $99.9 \%$, copper with $99.9 \%$, and titanium with $99.5 \%$. The preparation of nanometal fluids by EDM was performed in the dielectric fluid, which uses a discharge system to pulse power $T_{\mathrm{ON}}-T_{\mathrm{OFF}}$ in order to generate spark discharge between the electrodes of a very small space interval. The heat generated by EDM melts the surface of the electrode into metal particles or charged ions and then sputters and dissolves them in the dielectric fluid [14]. During the discharge machining process, a magnetic stirrer and stirring bar continuously operated at the bottom, thus allowing the nanoparticles in the fluid and temperature to be evenly distributed. The schematic diagram for preparing nanofluids using the electrical discharge method is as shown in Figure 1.

2.2. Dielectric Liquid. Discharge machining fluid (dielectric fluid) affects the degree of insulation of EDM at work. If the insulation of the dielectric fluid is poor, even if the electrode gap is considerably wide, it would break the insulation and discharge. When processing liquid contains conductive particles, it results in a decline of insulation in the processing liquid and causes an increased electrode gap. Different dielectric fluids [19] directly affect the electrode material removal rate (MRR), surface roughness [20], and electrode loss rate. Choosing pure dielectric fluid can help the preservation of nanofluids and convenience of analysis. If the dielectric fluid has suspended particles, it indirectly causes decreased surface roughness, thus, the surface to be machined must be relatively smoother. In other words, the amount of melted processing electrode by a single arc decreases and then lowers the removal rate of the material to be machined. However, if there are no suspended particles in the dielectric fluid, at the beginning of discharge, the resulting electrode discharge surface is processed roughly in order to generate many sophisticated salient points, thus causing endpoint discharge to lead to an increased material removal rate [21]. This study selected DW as the dielectric fluid. After

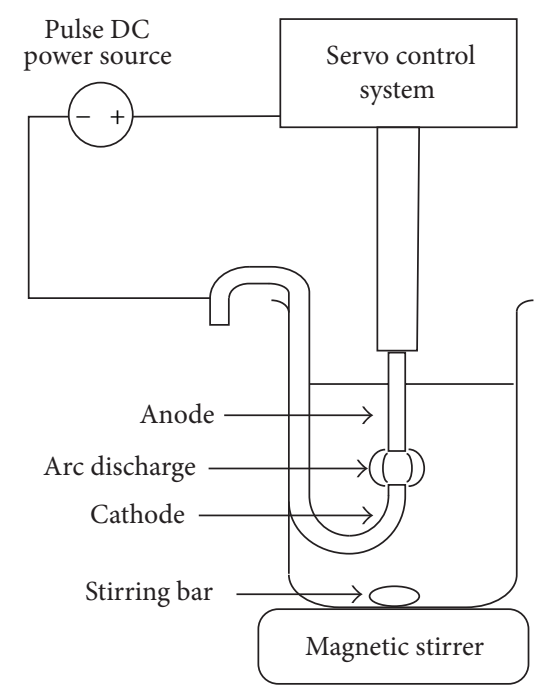

FIGURE 1: Schematic diagram of preparing nanofluids using the electrical discharge method.

electrical discharge machining, the molten metal particles from electrode and ions processing were suspended in the dielectric fluid.

2.3. Discharge Duration and Duty. In EDM machining processing, there are many discharge scenarios, including successful discharges, discharge difficulties, short circuit, and open circuit. Moreover, when changing the $T_{\mathrm{ON}}-T_{\mathrm{OFF}}$ period, $I_{P}$ (current), or adding capacitance settings to turn on the $Z$-axis settings, discharge waveform changes of EDM may occur, thus indirectly affecting the metallic properties of the fluid. During the discharge process of EDM, the power frequency provided by the system is very high, and discharge frequency ranges between $1 \mathrm{kHz} \sim 1 \mathrm{MHz}$ (period is $1 \mathrm{~ms} \sim$ $1 \mu \mathrm{s})$. If the discharge conditions are good, the servo control system maintains electrode spacing of about $30 \mu \mathrm{m}$, due to the stable current feedback signals. However, when the electrode spacing is too large, difficulties in arc discharge may result. When the $Z$-axis begins operation, the electrode spacing changes become more obvious. Figure 2 shows a schematic diagram of the discharge waveform.

2.4. Parameters Setting. EDM has many control parameters; however, during the preparation process of nanofluids, the main control parameters are as follows: (a) discharge pulse on-off duration ( $\left.T_{\mathrm{ON}}-T_{\mathrm{OFF}}\right)$ for setting the time of discharge $\left(T_{\mathrm{ON}}\right)$ and stop $\left(T_{\mathrm{OFF}}\right)$, which affects the accuracy of the EDM electrode consumption rate; (b) current $I_{P}$ setting, if the $I_{P}$ is greater, the discharge current is also greater and the processing speed is faster; however, it lowers the accuracy rate, resulting in electrode surface roughness, and the general finishing uses smaller $I_{P}$ settings; (c) capacitance value setting (capacitor) affects the instantaneous current at the state of discharge pulse $T_{\mathrm{ON}}$ and is suitable for materials of poor conductivity and discharge difficulties; however, in this study, adding the capacitance may cause poor precision 


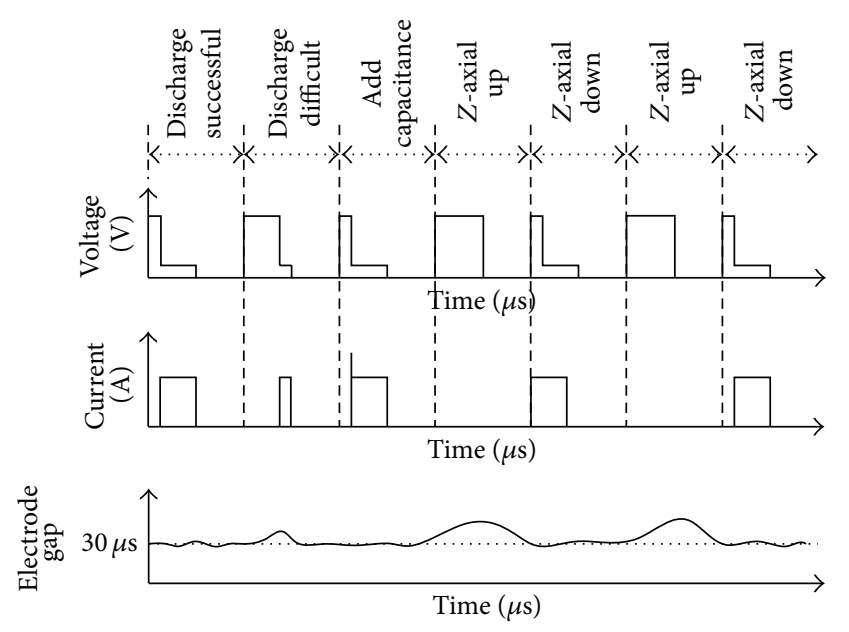

FIgURE 2: Schematic diagram with considerations of discharge difficulties, capacitor settings, and $Z$-axis settings.

of materials and many deposits at the bottom of the prepared nanofluids; (d) the vertical control knob (machining $Z$-axis) controls the up and down time of the $Z$-axis. If the setting is turned off, EDM can continuously process the anode; (e) high voltage switch (HV), the switch for the power supplies of $120 \mathrm{~V}$ and $240 \mathrm{~V}$. The experiment is carried under the normal temperature which is $25 \pm 1^{\circ} \mathrm{C}$.

\section{Results and Discussion}

3.1. Characterization of Silver Particles. The silver fluid with geometric $T_{\mathrm{ON}}-T_{\mathrm{OFF}}$ setting had the same UV absorption peak at $398 \mathrm{~nm}$, as shown in Figure 3. The silver ion absorption peak was $198 \mathrm{~nm}$, the particle size distribution was of approximately $1 \sim 10 \mathrm{~nm}$, and an improved material removal rate was obtained at $30: 30$ because it can get the highest absorption. The concentration of silver fluid declined with increasing proportions. As shown in Figure 3(b), when the $T_{\text {ON }}$ parameter was set, the UV absorption peak resulted in a red shift to $404 \mathrm{~nm}$ and the particle size distribution range became slightly wider. With the $T_{\mathrm{ON}}$ setting, the concentration of silver fluid declined. When the $T_{\mathrm{OFF}}$ parameter was set, the UV absorption peak resulted in a red shift to $404 \mathrm{~nm}$, and concentration declined with the setting of $T_{\mathrm{OFF}}$. However, the setting of $30: 50$ could obtain the best silver fluid concentration and smaller silver particles (particle size less than $1 \mathrm{~nm})$.

As shown in Figure 4, silver particles' Zeta-Potential was in the range of -30 to $-50 \mathrm{mV}$. Under the condition of no surface active agent, it still achieved good suspension stability. The fluid color changed according to particle size. The color was partially orange in the case of small particles, as compared to silver in the case of large particles. In an environment with an applied electric field, the dissociation of silver ions was exacerbated. When the concentration of charged ions increased, the DW degree of insulation declined, resulting in a higher success rate of electrode discharge. Discharge concentration led to rough and larger particle size;
TABLE 1: Nanosilver, EDX analysis of element proportion.

\begin{tabular}{lc}
\hline Element & Proportion (\%) \\
\hline $\mathrm{Ag}$ & 83.28 \\
$\mathrm{C}$ & 4.30 \\
$\mathrm{O}$ & 2.26 \\
$\mathrm{Nb}$ & 3.77 \\
$\mathrm{Cl}$ & 4.04 \\
$\mathrm{Al}$ & 2.35 \\
\hline
\end{tabular}

hence, the appropriate turnoff time $\left(T_{\mathrm{OFF}}\right)$ allowed the silver ions to be more smoothly discharged between the electrodes, in order to generate nanosilver fluids with high concentration and small particle size.

In SEM analysis, carbon gel was used as a carrier, where oxygen is accompanied by carbon glue, and $\mathrm{C}$ and $\mathrm{O}$ are found in the analysis. The SEM-EDX analysis results are as shown in Table 1 and Figure 5. EDX analysis had measurement of trace elements. As shown in Table 1, Nb, Cl, and $\mathrm{Al}$ in silver fluid were minor elements in the analysis.

3.2. Characterization of Copper Particles. Copper particles are shaped like rice grains in the SEM photograph, and most particle sizes are about 100 200 $\mathrm{nm}$. Under the setting parameters of all ratios of $T_{\mathrm{ON}}-T_{\mathrm{OFF}}$, various samples have the same UV absorption peaks of approximately $278 \mathrm{~nm}$, as shown in Figure 6. The fluid was copper yellow and did not change with the different settings of $T_{\mathrm{ON}}-T_{\mathrm{OFF}}$. The particle size results of zeta-size analysis, as shown in Figure 7, suggested that the copper particle size distribution is similar. Hence, the change of particle size can only be realized by changing the current $\left(I_{P}\right)$ of the process parameters.

Among the geometric parameters, the 10:10 setting resulted in better material removal rate. The concentration of the copper particles in the fluid was relatively high, and copper fluid concentration gradually decreased with rising proportions. The Zeta-Potential was concentrated around $-30 \mathrm{mV}$, indicating good suspension stability. When the parameter setting of $T_{\mathrm{ON}}$ increased, in a given process time, the copper fluid also increased with the increasing $T_{\mathrm{ON}}$, and the Zeta-Potential of copper particles was evenly distributed. For example, at a setting of $300: 10$, the Zeta-Potential was approximately $-30 \mathrm{mV}$ to achieve good suspension stability. When the $T_{\text {OFF }}$ parameter setting increased, the copper fluid concentration decreased with $T_{\mathrm{OFF}}$. By reducing working frequency and increasing turnoff time, the copper fluid concentration fell accordingly. The Zeta-Potential of copper particles was smaller and close to $-20 \mathrm{mV}$. Therefore, suspension stability was poor and resulted in deposition after one day. It is inferred that the times of successful discharges decreased due to lowering working frequency. As the ion concentration decreased due to discharge, the particle electric double layer was affected, resulting in a lower measurement of Zeta-Potential.

In EDX analysis, due to carbon glue, the analysis results of the copper fluid may have minor elements of $\mathrm{Ta}, \mathrm{Nb}$, and $\mathrm{Zr}$. The proportion of $\mathrm{C}$ and $\mathrm{O}$ in carbon glue was 


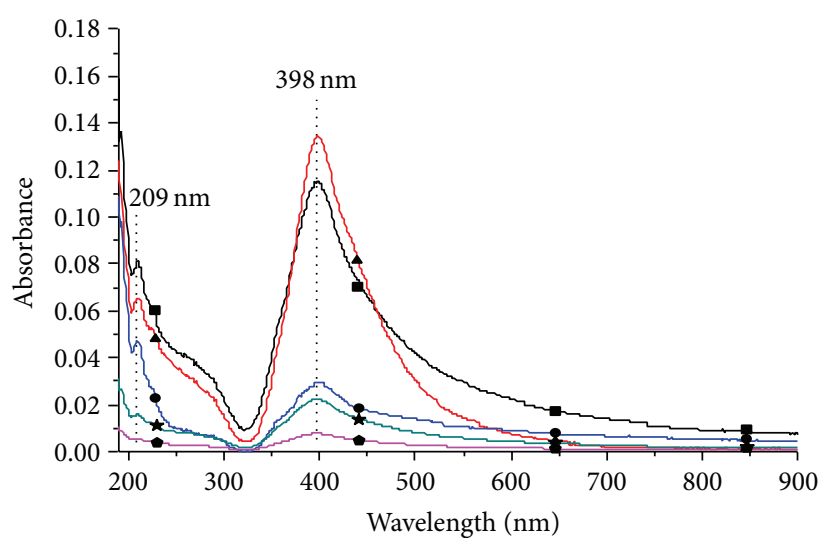

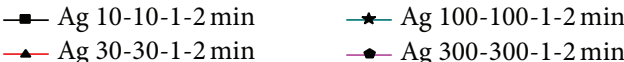
$\rightarrow$ Ag 50-50-1-2 min

(a)

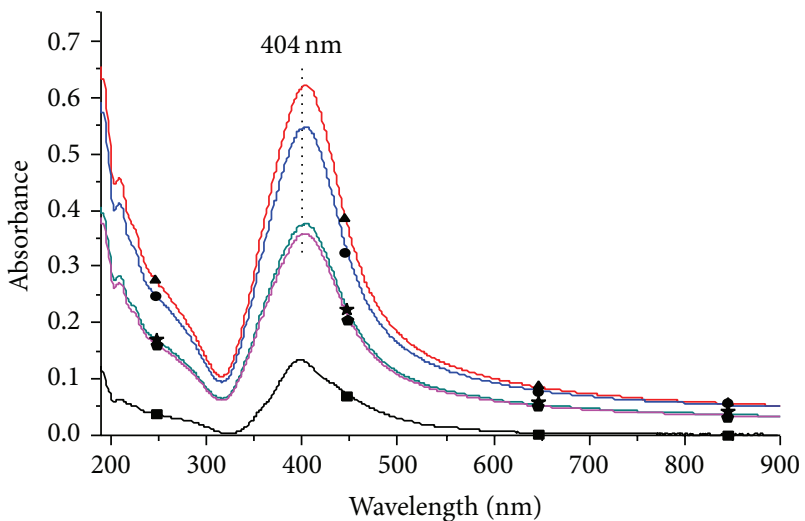

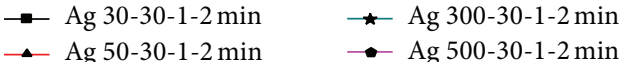
$\rightarrow$ Ag 100-30-1-2 min

(b)

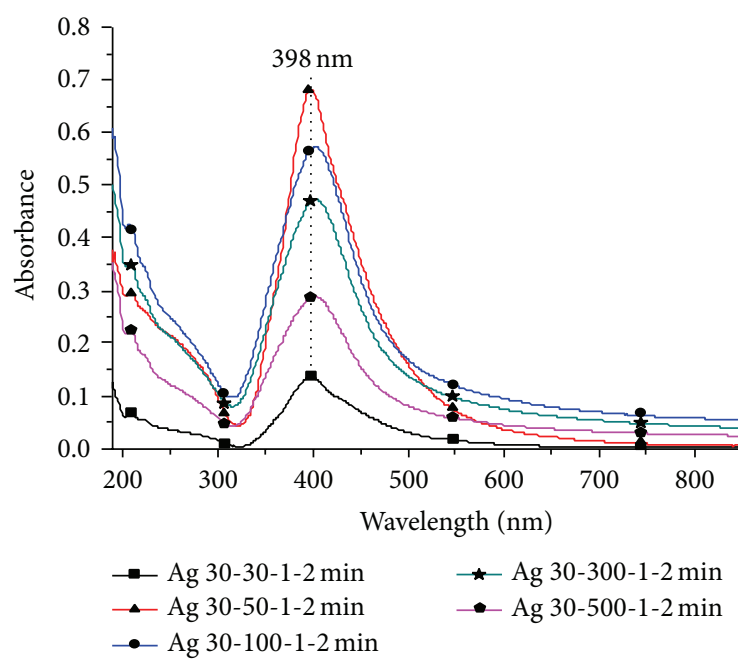

(c)

FIGURE 3: Silver fluid UV-V analysis results: (a) $T_{\mathrm{ON}}-T_{\mathrm{OFF}}$ in geometric proportion; (b) $T_{\mathrm{ON}}$ in progressive increase; (c) $T_{\mathrm{OFF}}$ in progressive increase.

TABLE 2: Nanocopper, EDX analysis of element proportion.

\begin{tabular}{lc}
\hline Element & Proportion (\%) \\
\hline $\mathrm{Cu}$ & 41.13 \\
$\mathrm{C}$ & 27.30 \\
$\mathrm{O}$ & 27.39 \\
$\mathrm{Ta}$ & 2.62 \\
$\mathrm{Nb}$ & 0.56 \\
$\mathrm{Zr}$ & 1.00 \\
\hline
\end{tabular}

about $9: 1$; therefore, about $41.13 \% \mathrm{C}$ was accompanied by $4.57 \% \mathrm{O}$, and copper in the fluid seemed to react with oxygen during the discharge process to generate the copperoxygen compound. The copper particle may be $\mathrm{CuO}$. Figure 8 and Table 2 illustrate the SEM and EDX analysis results of nanocopper particles.
3.3. Characterization of Titanium Particles. Under the settings of all proportions of $T_{\mathrm{ON}}-T_{\mathrm{OFF}}$, nanotitanium fluid had the same UV absorption peak of approximately $245 \mathrm{~nm}$, as shown in Figure 9. Without obvious wavelength shift, the particle size distribution of titanium particles was observed as very similar in the zeta-size analysis. Particle size was distributed in the case of three groups of parameters of approximately $100 \mathrm{~nm}$, as shown in Figure 10. The optimal process parameters of titanium fluid are $T_{\mathrm{ON}}$ set at $100 \mu \mathrm{s}$ and $T_{\mathrm{ON}}$ set as greater or lower than $100 \mu \mathrm{s}$, and the titanium fluid concentration gradually decreased. When the $T_{\mathrm{OFF}}$ was greater, the turnoff time was longer, the concentration of titanium particles was lower, and the color of the titanium fluid was uniformly dark blue with fluorescent colors. Under the condition of a given current, the characteristics of the titanium fluid by the changes in $T_{\mathrm{ON}}-T_{\mathrm{OFF}}$ parameter settings were similar to copper. In other words, the distribution of particles had no significant difference. Changing particle size 


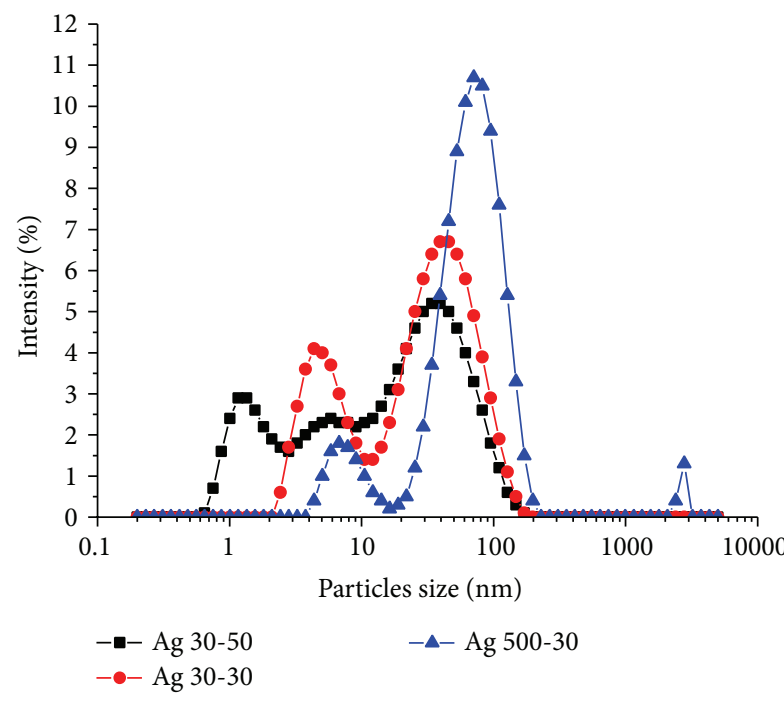

(a)

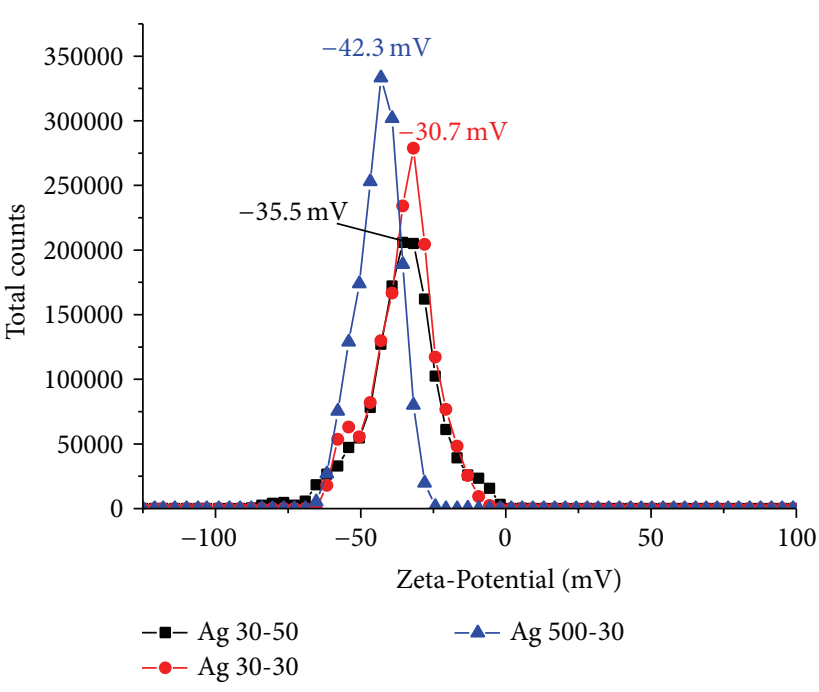

(b)

FIgURe 4: Nanosilver fluid. (a) Particles size analysis; (b) Zeta-Potential analysis.
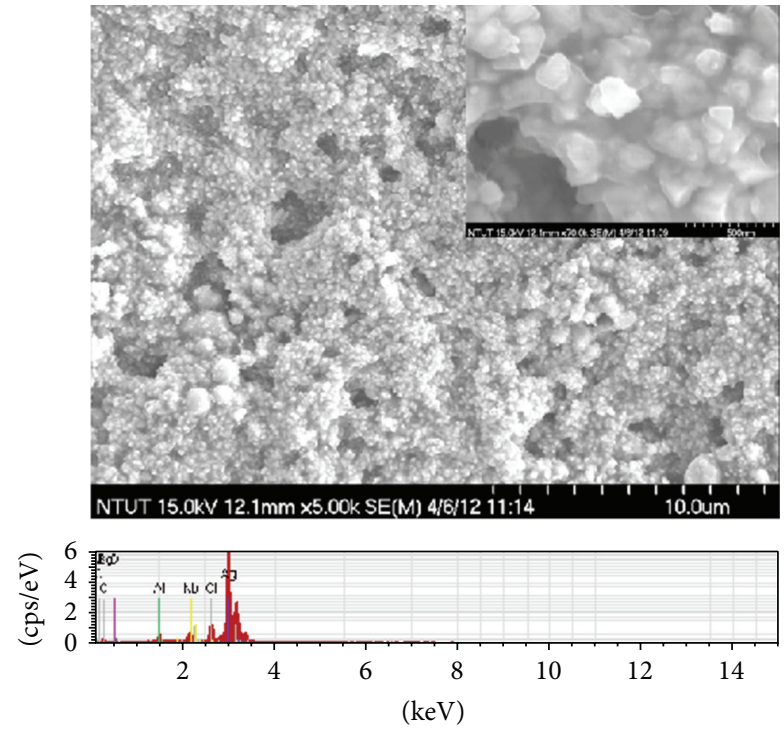

FIgURE 5: Nano silver, SEM (SEM-EDX).

was realized by current $\left(I_{P}\right)$ setting, controlled discharge temperature, or adding surface active agent. Under the given current condition, the Zeta-Potential of titanium particles of various proportions of $T_{\mathrm{ON}}-T_{\mathrm{OFF}}$ was similar. The ZetaPotential was negative at $-40 \mathrm{mV}$, thus, the suspension characteristics were good.

To sum up, the characteristics of titanium particles prepared in the case of various $T_{\mathrm{ON}}-T_{\mathrm{OFF}}$ proportions were similar; however, the concentration of the fluid varied. The hardness of titanium material was very high, and its resistivity was relatively higher than other metals. It can be observed from the settings of discharge that better fluid concentration can only be obtained by a longer $T_{\mathrm{ON}}$ setting. Under the conditions of medium and high frequency ( $\mu$ s grade),
TABLE 3: Nanotitanium, EDX analysis of element proportion.

\begin{tabular}{lc}
\hline Element & Proportion (\%) \\
\hline $\mathrm{Ti}$ & 50.95 \\
$\mathrm{C}$ & 6.21 \\
$\mathrm{O}$ & 35.92 \\
$\mathrm{Au}$ & 5.60 \\
$\mathrm{Al}$ & 1.32 \\
\hline
\end{tabular}

the resistivity of metal electrodes affects the discharge of the process.

SEM-EDX analysis results of the titanium fluid are as shown in Table 3. Besides the minor elements ( $\mathrm{Au}$ and $\mathrm{Al}$ ), it can be found that oxygen accounts for about $1 / 3$ of the particle. The analysis results are similar to those of the copper articles. It can be concluded that titanium reacts with oxygen during the discharge process to generate compounds, and the product of the fluid is very likely to be $\mathrm{TiO}_{2}$ [22]. SEM analysis results are as shown in Figure 11.

3.4. Results and Discussion. When using EDM electrosparking for the preparation of fluid, the distribution of metal particles in the fluid was very wide. Unlike the chemical method to accurately control particle size, this method can produce large amounts with high efficiency. Metals have different characteristics. The results indicated that metal characteristics have great influence on the preparation of metal fluids using the electrosparking method. The temperature of arc is very high in order to melt the metal and vaporize it into nanometer particles. The discharge frequency is very high and is similar to a pulse discharge. In the environment of DW discharge, the conductivity of metal materials affects the sparking discharge, and the characteristics of the prepared fluids differ accordingly. The resistivity of silver and copper 


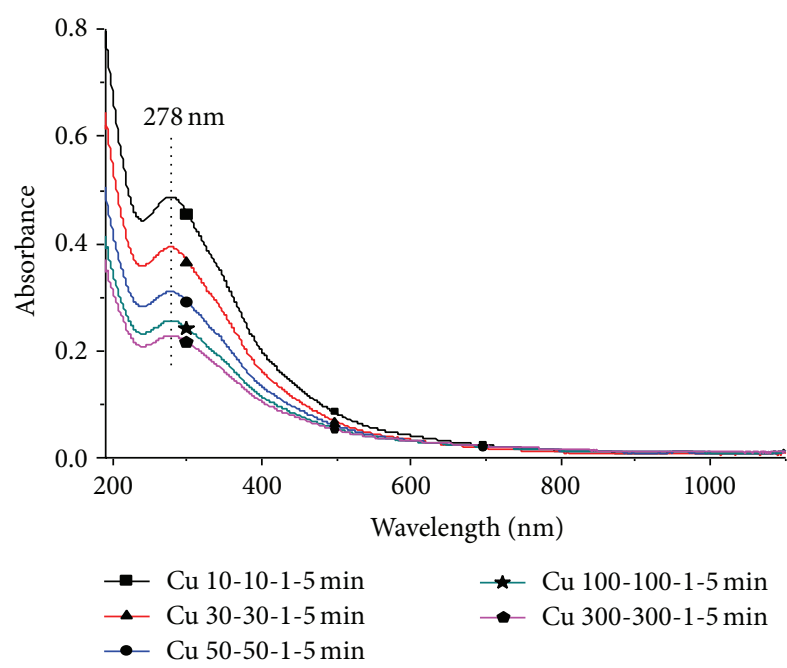

(a)

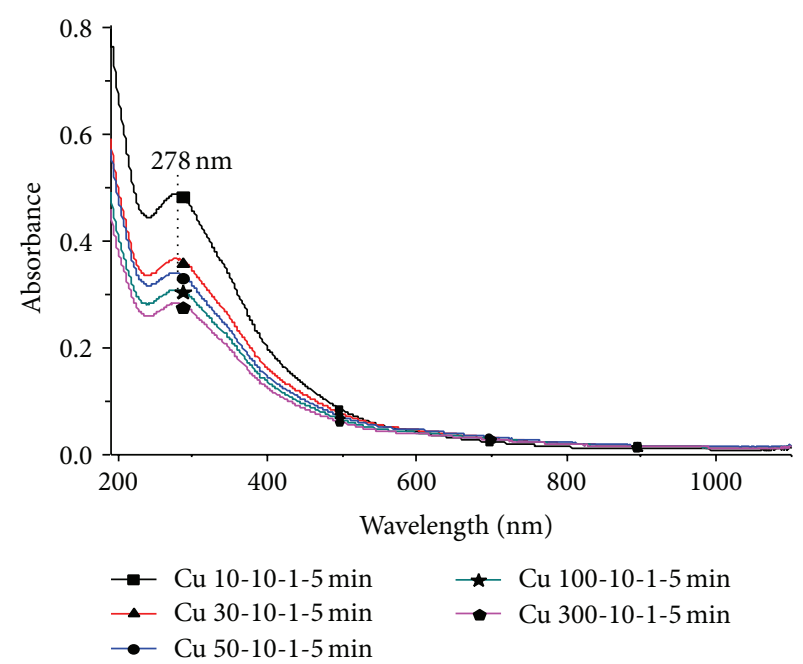

(b)

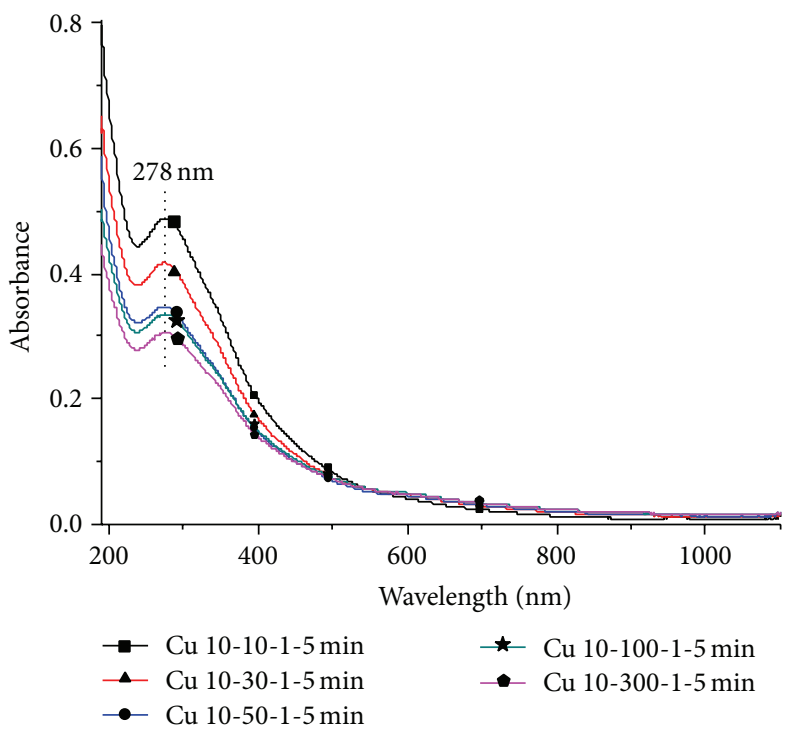

(c)

FIGURE 6: Copper fluid UV-Vis analysis results; (a) $T_{\mathrm{ON}}-T_{\mathrm{OFF}}$ in geometric proportion; (b) $T_{\mathrm{ON}}$ in progressive increase; (c) $T_{\mathrm{OFF}}$ in progressive increase.

is very low, at about $15 \times 10^{-9} \Omega \cdot \mathrm{m}$. If $T_{\mathrm{ON}}$ is set in the range of $10 \sim 50 \mu \mathrm{s}$, a good discharge success rate could be obtained, and the titanium resistivity is $420 \times 10^{-9} \Omega \cdot \mathrm{m}$, which is far greater than silver or copper. Hence, a good discharge success rate can be obtained when $T_{\mathrm{ON}}$ is set at approximately $100 \mu \mathrm{s}$.

This study found that pulse setting $\left(T_{\mathrm{ON}}-T_{\mathrm{OFF}}\right)$ mainly affects fluid concentration. However, the current setting and ambient temperature affect particle size, while $T_{\mathrm{ON}}-T_{\mathrm{OFF}}$ affects particle size distribution range. Silver has obvious UV shifts with $T_{\mathrm{ON}}-T_{\mathrm{OFF}}$ settings. The minimum particle distribution can be obtained at the proportion of $30: 50$. The UV absorption wavelength shift is not apparent in the case of copper and titanium, as compared with silver. In the particle size distribution analysis, it can be confirmed that the particle size distribution of copper and silver has no significant difference in the various settings of $T_{\mathrm{ON}}-T_{\mathrm{OFF}}$.
The results of UV-Vis analysis suggest that fluid concentration changes with $T_{\mathrm{ON}}-T_{\mathrm{OFF}}$ settings. In zeta-size analysis, the distribution of various metal particles in DW can be analyzed. It is confirmed that the Zeta-Potential can be maintained around $-30 \mathrm{mV}$ without adding a surface active agent, indicating that the particles in the fluid have very good suspension stability. Zeta-Potential changes with different settings of $T_{\mathrm{ON}}-T_{\mathrm{OFF}}$. The particle distribution peak value and Zeta-Potential peak value of metals, in the cases of different discharge parameters, are as shown in Table 4.

\section{Conclusions}

This study discussed the parameters of three common metals, including silver and copper with good electric and thermal conductivity, and titanium with high toughness. This study 


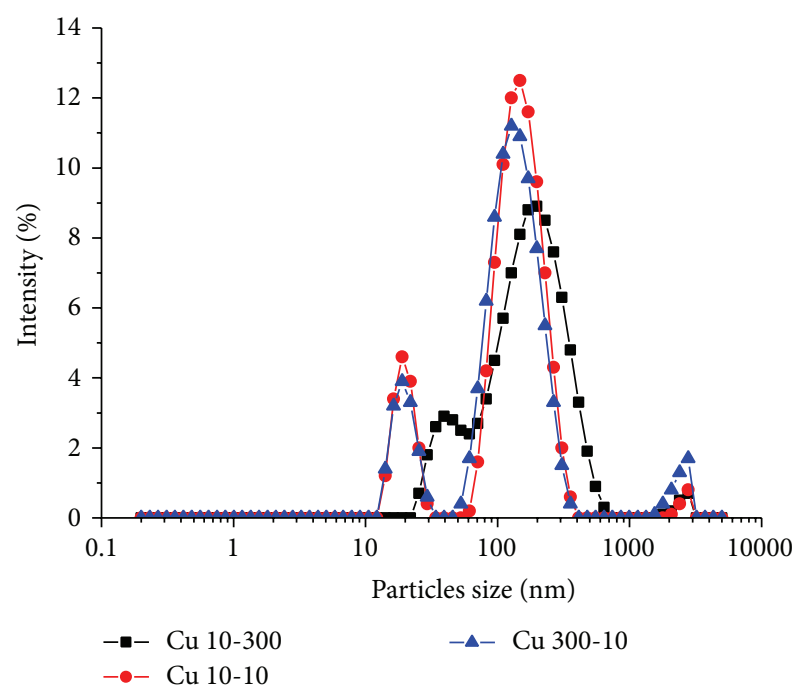

(a)

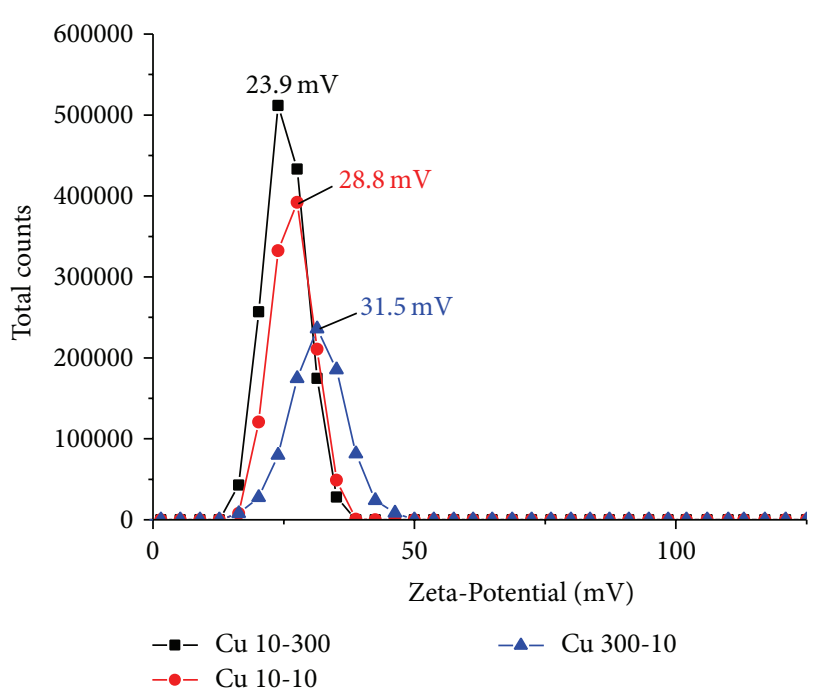

(b)

FIgure 7: Nanocopper fluid. (a) Particles size analysis; (b) Zeta-Potential analysis.
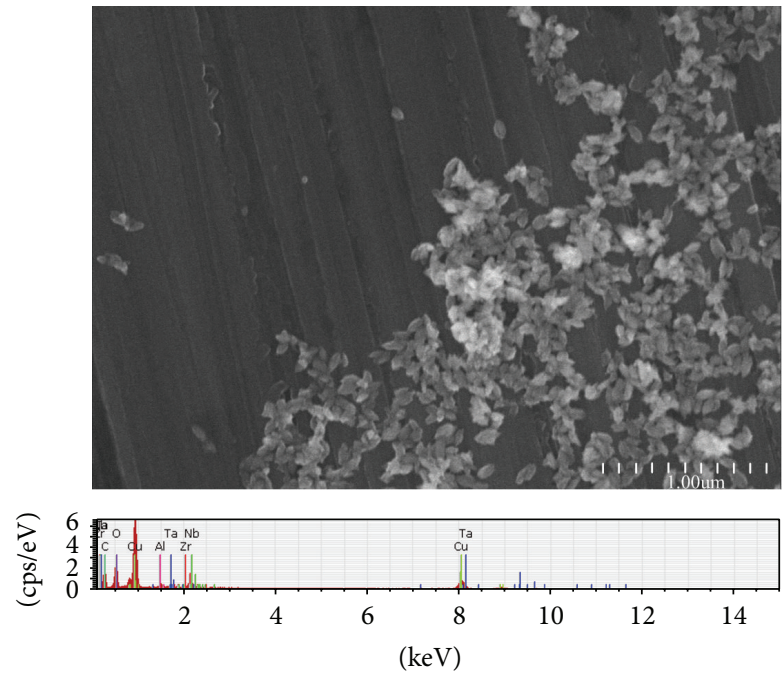

FIgURE 8: Nanocopper, SEM (SEM-EDX).

explored the changes of the characteristics of the nanofluids of various metals in cases of different proportions of $T_{\mathrm{ON}^{-}}$ $T_{\mathrm{OFF}}$. The parameter settings were discussed according to parameter settings of geometric proportion, increased discharge time, and increased turnoff time, in order to summarize the law between parameters and fluid characteristics. Based on the current findings, our future study will attempt to plan electrosparking process parameters that can achieve the optimum process efficiency for the mass production of nanofluids according to the metal properties.

The conclusions of this study are as follows.

(1) In an environment of room temperature and atmospheric pressure, metal nanofluids were prepared
TABLe 4: Particle distribution peak value and Zeta-Potential peak value of metals in the case of different discharge parameters.

\begin{tabular}{lccc}
\hline Material & $\begin{array}{c}\text { Discharge } \\
\text { parameters }\end{array}$ & $\begin{array}{c}\text { Particle } \\
\text { distribution } \\
\text { peak value }\end{array}$ & $\begin{array}{c}\text { Zeta-Potential } \\
\text { peak value }\end{array}$ \\
\hline \multirow{3}{*}{ Silver } & $30: 50$ & $40 \mathrm{~nm}$ & $-35 \mathrm{mV}$ \\
& $30: 30$ & $38 \mathrm{~nm}$ & $-32 \mathrm{mV}$ \\
& $500: 30$ & $41 \mathrm{~nm}$ & $-43 \mathrm{mV}$ \\
\hline \multirow{3}{*}{ Copper } & $10: 300$ & $198 \mathrm{~nm}$ & $-24 \mathrm{mV}$ \\
& $10: 10$ & $148 \mathrm{~nm}$ & $-28 \mathrm{mV}$ \\
Titanium & $300: 10$ & $128 \mathrm{~nm}$ & $-32 \mathrm{mV}$ \\
& $10: 300$ & $110 \mathrm{~nm}$ & $-42 \mathrm{mV}$ \\
& $10: 10$ & $128 \mathrm{~nm}$ & $-43 \mathrm{mV}$ \\
& $300: 10$ & $128 \mathrm{~nm}$ & $-40 \mathrm{mV}$ \\
\hline
\end{tabular}

using electrosparking, and the particles stably suspended in DW without adding any surface suspending agent.

(2) Silver's resistivity was low $\left(15 \times 10^{-9} \Omega \cdot \mathrm{m}\right)$; thus, when the $T_{\mathrm{ON}}-T_{\mathrm{OFF}}$ ratio was set to $30: 50$. A nanosilver fluid with the best concentration and smallest particle size was prepared. In the electric field, the dissociation of silver was very good, and nanosilver fluids of high concentration and small particle size were easily prepared.

(3) In electrosparking of copper, current $\left(I_{P}\right)$ was set fixed, and in the case of various proportions of $T_{\mathrm{ON}^{-}}$ $T_{\mathrm{OFF}}$, the UV absorption peak and particle size distribution of the resulting copper fluids differed. Changes in fluid density and $T_{\mathrm{ON}}-T_{\mathrm{OFF}}$ were consistent, and the Zeta-Potential of copper particles was generally lower. 


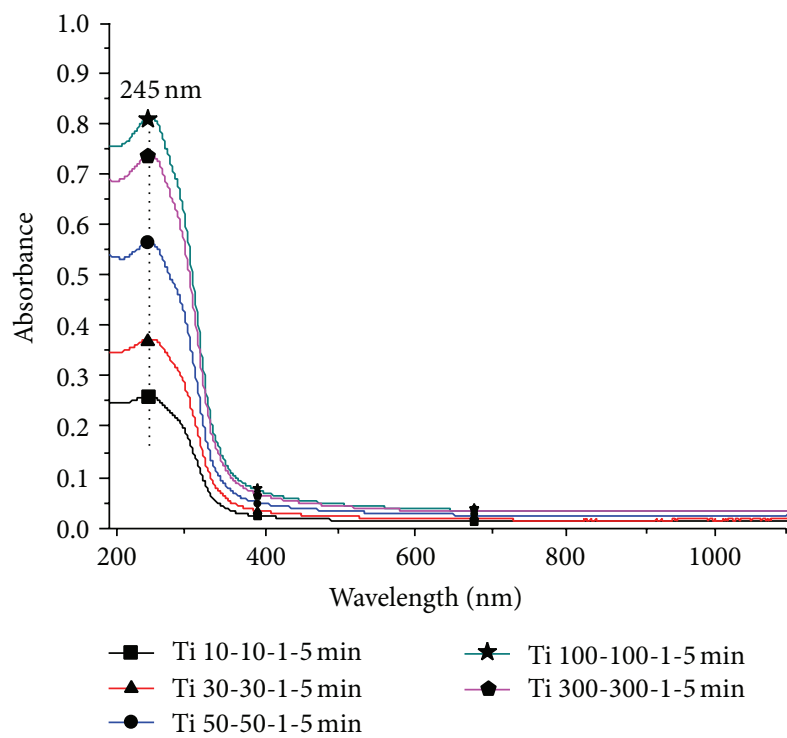

(a)

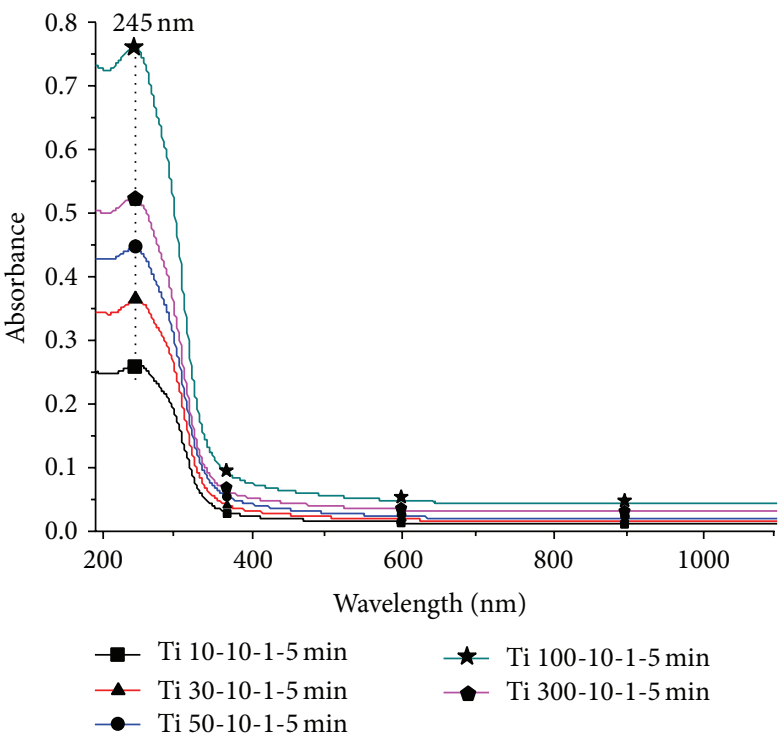

(b)

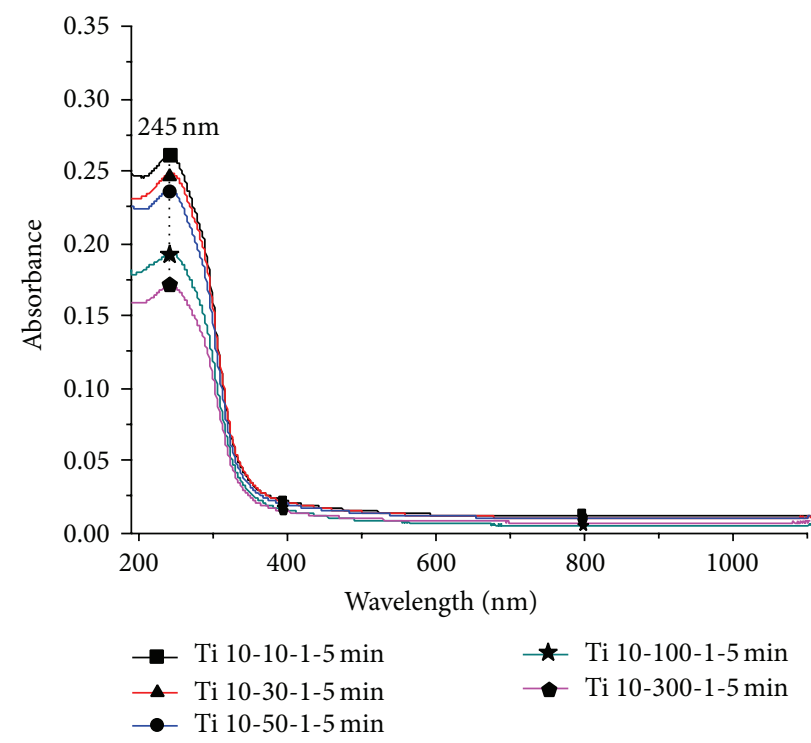

(c)

FigURE 9: Titanium fluid UV-Vis analysis results. (a) $T_{\mathrm{ON}}-T_{\mathrm{OFF}}$ in geometric proportion; (b) $T_{\mathrm{ON}}$ in progressive increase; (c) $T_{\mathrm{OFF}}$ in progressive increase.

(4) The resistivity of titanium was greater $(420 \times$ $\left.10^{-9} \Omega \cdot \mathrm{m}\right)$; thus, good discharge was obtained when the $T_{\mathrm{ON}}$ setting was $100 \mu \mathrm{s}$. The example of titanium suggested that it takes a longer discharge time to set the material removal rate in the case of metals with poor conductivity rates.

(5) The results of UV-Vis analysis suggested that the discharge pulse $\left(T_{\mathrm{ON}}-T_{\mathrm{OFF}}\right)$ setting is the main factor influencing fluid concentration. Zeta-Potential changed with different settings of $T_{\mathrm{ON}}-T_{\mathrm{OFF}}$ proportions. The analysis results of zeta-size indicated that without adding a surface active agent, the ZetaPotential could be maintained around $-30 \mathrm{mV}$, or better, indicating that the particles in the fluid have very good suspension stability.

(6) The oxygen and hydrogen are electrolyzed from the water when the discharge is happening. The metal will combine some of the oxygen. Besides, when analyzing the samples with SEM-EDX, the process of drying will also cause the oxidation phenomenon. The carbon came from the carbon conductive tape when doing the analysis of SEM-EDX. The minor elements will be 


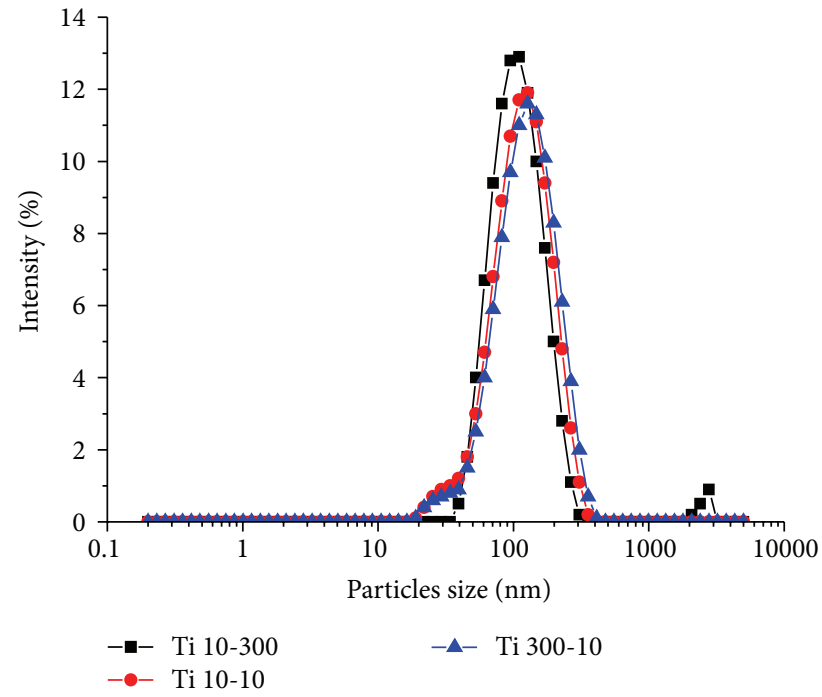

(a)

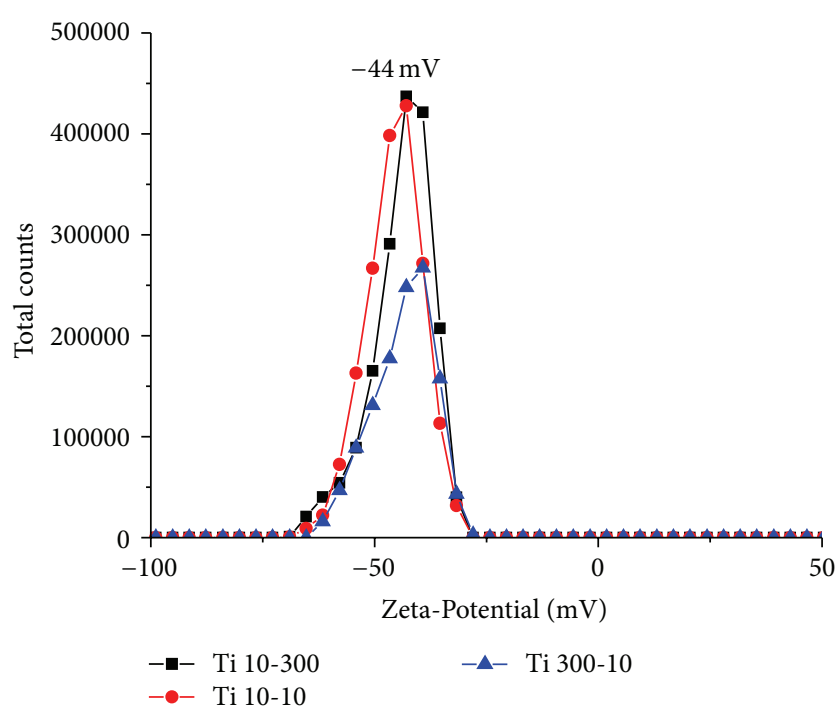

(b)

Figure 10: Nanotitanium fluid. (a) Particles size analysis; (b) Zeta-Potential analysis.
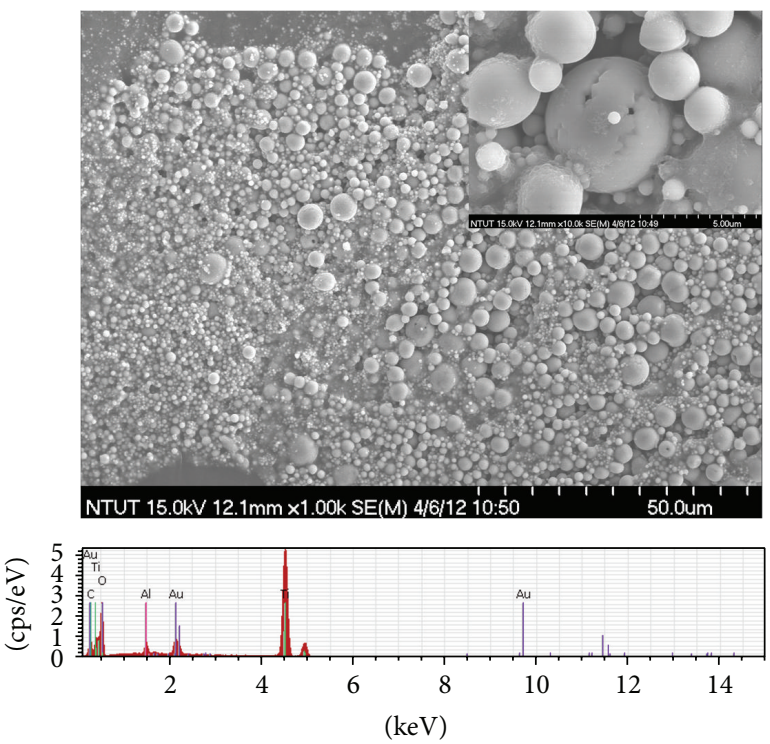

FIGURE 11: Nanotitanium, SEM (SEM-EDX).

detected because the purity of $\mathrm{Ag}, \mathrm{Cu}$, and $\mathrm{Ti}$ is only up to $99.9 \%$.

\section{Conflict of Interests}

The authors declare that there is no conflict of interests regarding the publication of this paper.

\section{Acknowledgment}

The authors would like to thank the National Science Council of the Republic of China (NSC 102-2221-E-027-035) for financially supporting this research.

\section{References}

[1] M. F. Meléndrez, G. Cárdenas, J. V. Díaz, C. C. Cruzat, and J. Arbiol, "Synthesis and aggregation study of tin nanoparticles and colloids obtained by chemical liquid deposition," Colloid and Polymer Science, vol. 287, no. 1, pp. 13-22, 2009.

[2] W. Yu, D. M. France, J. L. Routbort, and S. U. S. Choi, "Review and comparison of nanofluid thermal conductivity and heat transfer enhancements," Heat Transfer Engineering, vol. 29, no. 5, pp. 432-460, 2008.

[3] B. M. Schumacher, "After 60 years of EDM the discharge process remains still disputed," Journal of Materials Processing Technology, vol. 149, no. 1-3, pp. 376-381, 2004.

[4] J. Jiang, G. Oberdörster, and P. Biswas, "Characterization of size, surface charge, and agglomeration state of nanoparticle dispersions for toxicological studies," Journal of Nanoparticle Research, vol. 11, no. 1, pp. 77-89, 2009.

[5] S. Jailani, G. V. Franks, and T. W. Healy, " $\zeta$ potential of nanoparticle suspensions: effect of electrolyte concentration, particle size, and volume fraction," Journal of the American Ceramic Society, vol. 91, no. 4, pp. 1141-1147, 2008.

[6] H. Chang and M.-K. Liu, "Fabrication and process analysis of anatase type $\mathrm{TiO}_{2}$ nanofluid by an arc spray nanofluid synthesis system," Journal of Crystal Growth, vol. 304, no. 1, pp. 244-252, 2007.

[7] H. Chang, M.-J. Kao, C.-S. Jwo, C.-G. Kuo, Y.-H. Yeh, and W.C. Tzeng, "Preparation of $\mathrm{Co} / \mathrm{Ag}$ nanocompound fluid using ASNSS with aid of ultrasonic orthogonal vibration," Journal of Alloys and Compounds, vol. 504, upplement 1, pp. S376-S379, 2010.

[8] K.-H. Tseng, C.-Y. Liao, and D.-C. Tien, "Silver carbonate and stability in colloidal silver: a by-product of the electric spark discharge method," Journal of Alloys and Compounds, vol. 493, no. 1-2, pp. 438-440, 2010.

[9] D.-C. Tien, K.-H. Tseng, C.-Y. Liao, and T.-T. Tsung, "Colloidal silver fabrication using the spark discharge system and its antimicrobial effect on Staphylococcus aureus," Medical Engineering \& Physics, vol. 30, no. 8, pp. 948-952, 2008. 
[10] D.-C. Tien, K.-H. Tseng, C.-Y. Liao, J.-C. Huang, and T.T. Tsung, "Discovery of ionic silver in silver nanoparticle suspension fabricated by arc discharge method," Journal of Alloys and Compounds, vol. 463, no. 1-2, pp. 408-411, 2008.

[11] K.-H. Tseng and J.-C. Huang, "Pulsed spark-discharge assisted synthesis of colloidal gold nanoparticles in ethanol," Journal of Nanoparticle Research, vol. 13, no. 7, pp. 2963-2972, 2011.

[12] K.-H. Tseng, C.-Y. Liao, J.-C. Huang, D.-C. Tien, and T.-T. Tsung, "Characterization of gold nanoparticles in organic or inorganic medium (ethanol/water) fabricated by spark discharge method," Materials Letters, vol. 62, no. 19, pp. 3341-3344, 2008.

[13] C.-H. Lo, T.-T. Tsung, and L.-C. Chen, "Shape-controlled synthesis of Cu-based nanofluid using submerged arc nanoparticle synthesis system (SANSS)," Journal of Crystal Growth, vol. 277, no. 1-4, pp. 636-642, 2005.

[14] H. Chang, X.-Q. Chen, C.-S. Jwo, and S.-L. Chen, "Electrostatic and sterical stabilization of $\mathrm{CuO}$ nanofluid prepared by vacuum arc spray nanofluid synthesis system (ASNSS)," Materials Transactions, vol. 50, no. 8, pp. 2098-2103, 2009.

[15] H. Chang and Y.-C. Chang, "Fabrication of $\mathrm{Al}_{2} \mathrm{O} 3$ nanofluid by a plasma arc nanoparticles synthesis system," Journal of Materials Processing Technology, vol. 207, no. 1-3, pp. 193-199, 2008.

[16] K.-H. Tseng, Y.-C. Chen, and J.-J. Shyue, “Continuous synthesis of colloidal silver nanoparticles by electrochemical discharge in aqueous solutions," Journal of Nanoparticle Research, vol. 13, no. 5, pp. 1865-1872, 2011.

[17] S. H. Yeo, W. Kurnia, and P. C. Tan, "Critical assessment and numerical comparison of electro-thermal models in EDM," Journal of Materials Processing Technology, vol. 203, no. 1-3, pp. 241-251, 2008.

[18] K.-H. Tseng, H.-L. Lee, C.-Y. Liao, K.-C. Chen, and H.-S. Lin, "Rapid and efficient synthesis of silver nanofluid using electrical discharge machining," Journal of Nanomaterials, vol. 2013, Article ID 174939, 6 pages, 2013.

[19] F. N. Leão and I. R. Pashby, "A review on the use of environmentally-friendly dielectric fluids in electrical discharge machining," Journal of Materials Processing Technology, vol. 149, no. 1-3, pp. 341-346, 2004.

[20] K. Salonitis, A. Stournaras, P. Stavropoulos, and G. Chryssolouris, "Thermal modeling of the material removal rate and surface roughness for die-sinking EDM," International Journal of Advanced Manufacturing Technology, vol. 40, no. 3-4, pp. 316323, 2009.

[21] S. H. Yeo, P. C. Tan, and W. Kurnia, "Effects of powder additives suspended in dielectric on crater characteristics for micro electrical discharge machining," Journal of Micromechanics and Microengineering, vol. 17, no. 11, pp. N91-N98, 2007.

[22] H. Chang and S.-C. Lin, "Fabrication method for a $\mathrm{TiO}_{2}$ nanofluid with high roundness and superior dispersion properties," Materials Transactions, vol. 48, no. 4, pp. 836-841, 2007. 

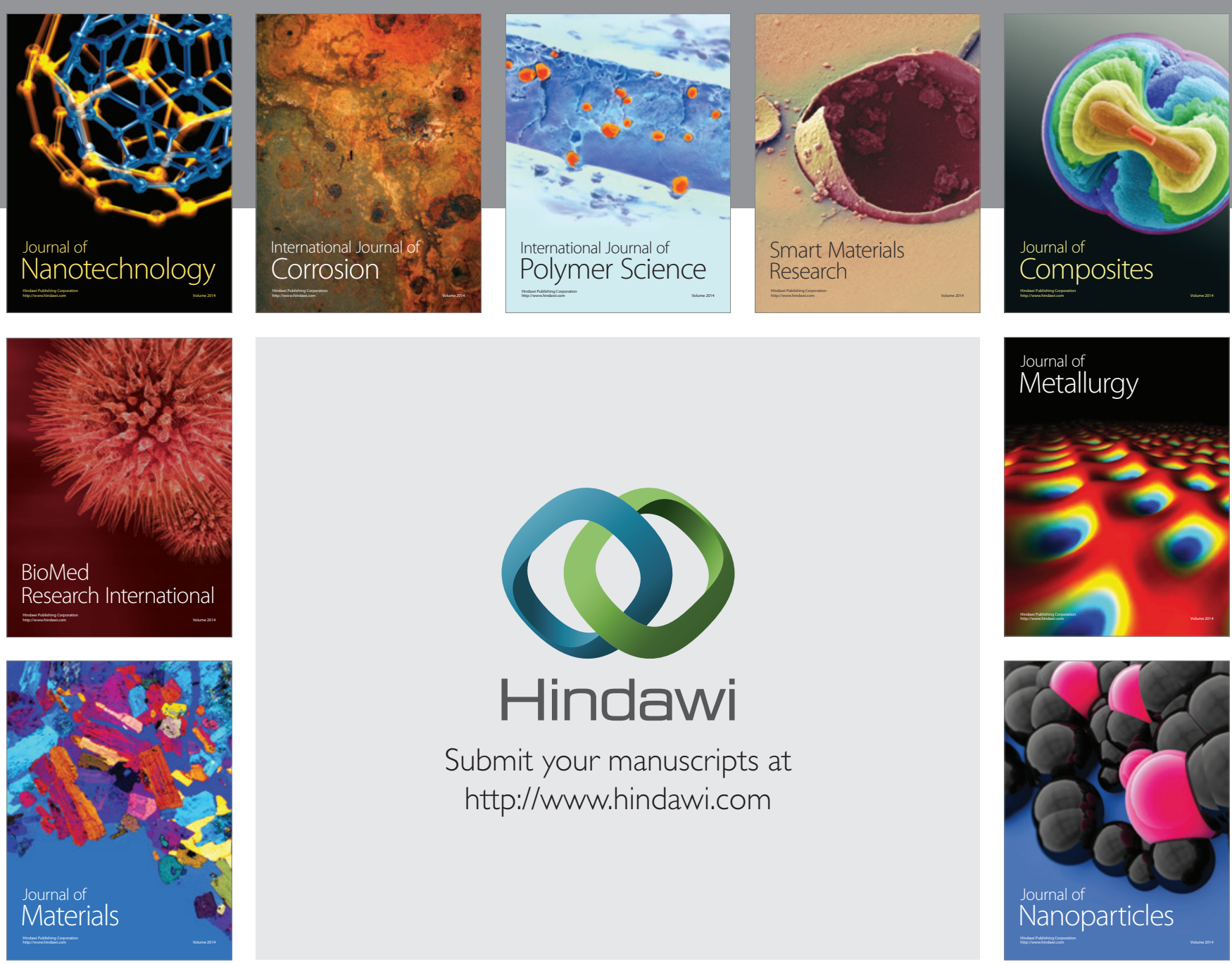

Submit your manuscripts at http://www.hindawi.com
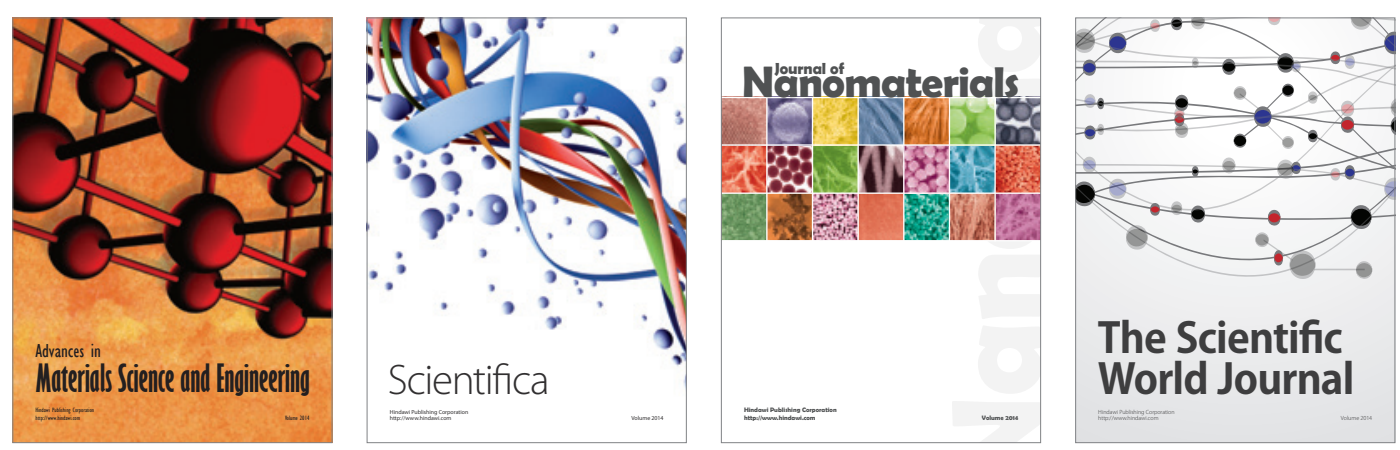

\section{The Scientific World Journal}
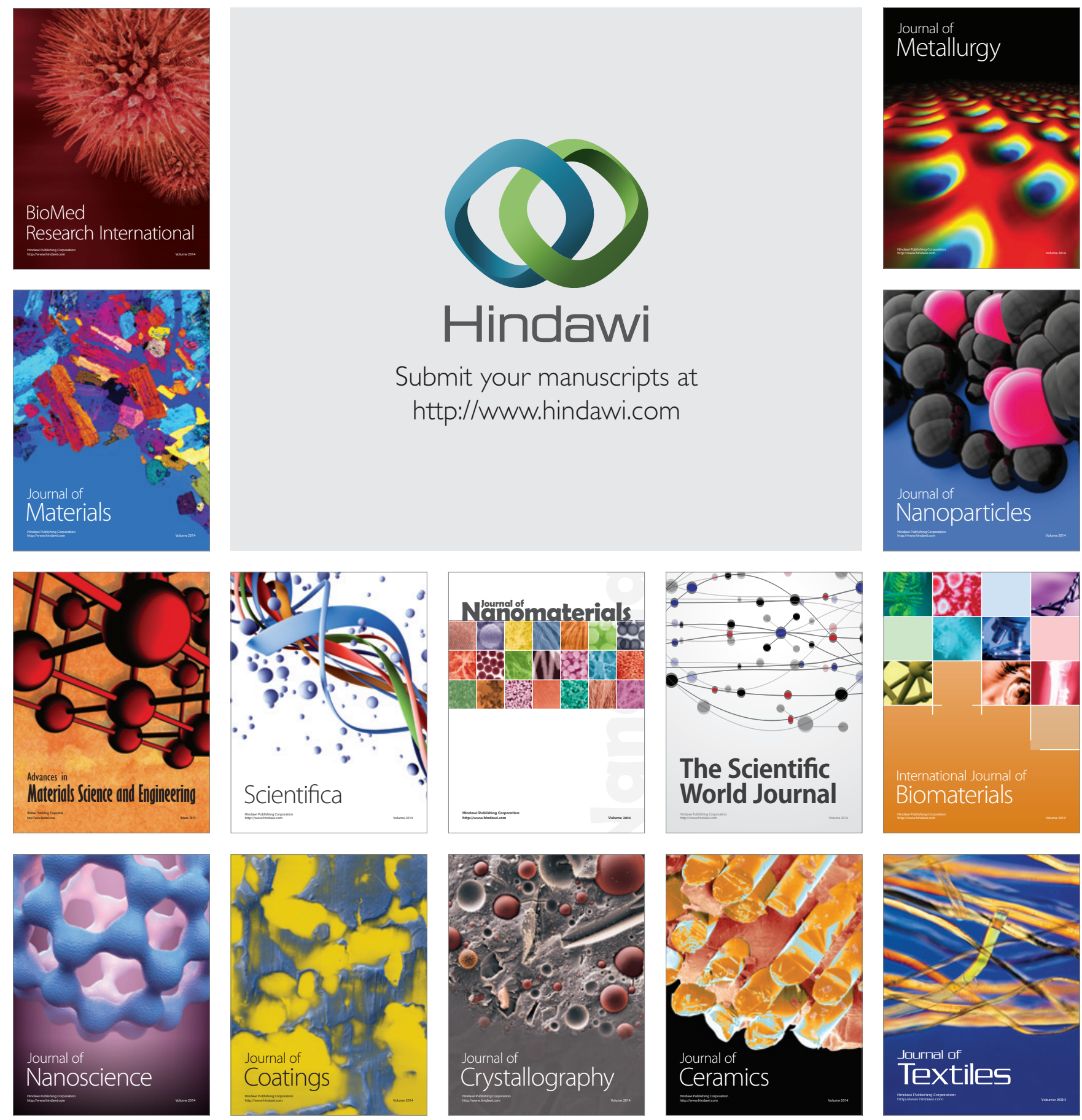